\title{
Review on Mechanical property Modification technique of Polyetheretherketone (PEEK) Composites
}

\author{
Dey Sujoy Kumar ${ }^{1}$, Mainali Vikash ${ }^{2}$, and Samanta Sutanu ${ }^{3}$
}

\begin{abstract}
Polyetheretherketone (PEEK) is one of the popular thermoplastic polymer due to its mechanical properties. The durability is very high under high temperature condition. Various modification have been done in PEEK matrix by incorporating different ceramics like $\mathrm{SiC}, \mathrm{SiO}_{2}, \mathrm{AlN}$, Zirconia, hydroxyapatite (HA), $\mathrm{Al}_{2} \mathrm{O}_{3}$, addition of fibers like glass, carbon fibers, PTFE and its effects in the properties have been studied. Properties changes are made in the PEEK to increase its utility in tribological field and application in medical. This review summarizes the effect of the inclusion of the various materials and its effect on hardness, wear rate, tensile strength of PEEK.
\end{abstract}

Keywords---Polyetheretherketone, modifications, mechanical Properties

\section{INTRODUCTION}

$\mathrm{P}$ OLYETHERETHERKETONE is a semi crystalline thermoplastics having a melting point $\left(\mathrm{T}_{\mathrm{m}}=335^{\circ} \mathrm{C}\right)$ and glass transition temperature $\left(\mathrm{T}_{\mathrm{g}}=143^{\circ} \mathrm{C}\right)$. PEEK has found its applications in aerospace industries, manufacturing industries due to its resistance to chemical, showing stability at very high temperatures, toughness [1]. The Coefficient of friction of this material is around 0.7 which is very high for its use as an anti-friction. Various types of modifications has been done in order to change the mechanical properties of PEEK, to increase its performance in different condition like high load, dry condition, lubricating. In this review paper addition of different reinforcements of fiber, mixing of nano/micro particles in the PEEK and its effects in the properties of PEEK has been discussed.

Sujoy Kumar Dey ${ }^{1}$, Assistant professor of Mechanical Engineering Department, Sikkim Manipal Institute of Technology, Sikkim-737132.

Vikash Mainali ${ }^{2}$, Post graduate student of Mechanical Engineering Department, Sikkim Manipal Institute of Technology, Sikkim-737132.

Sutanu Samanta ${ }^{3}$, Associate Professor of Mechanical Engineering Department, North Eastern Regional Institute of Science and Technology, Nirjuli, Arunachal Pradesh-791109.

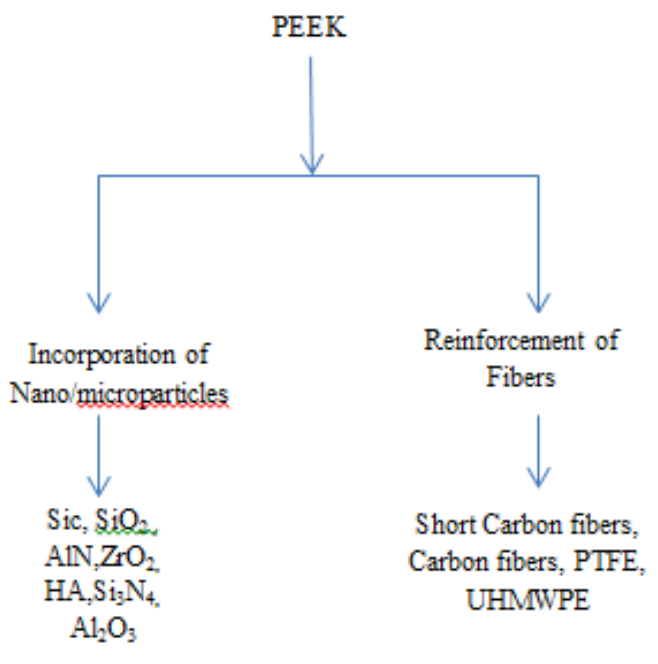

\section{MODIFICATION TECHNIQUE}

PEEK has been modified using following techniques, (a) Reinforcement of fiber \& (b) mixing of micro/nano particles. It has been seen that incorporation of particulate is easier then reinforcing fibers into the PEEK. The PEEK powders are mixed with nano/micro particles in an alcohol medium then dried and sintered [2].

It has been seen that incorporation of particulate is easier then reinforcing fibers into the PEEK. The PEEK powders are mixed with nano/micro particles in an alcohol medium then dried and sintered [2].

\section{DISCUSSIONS}

When PEEK composites are formed with $\mathrm{SiC}$, it is found that coefficient of friction reduces and even the wear rate decreases for certain ratio of mixing between $7.5 \mathrm{wt} \%$ to 10 wt. \%. SiC fillers are found to efficient in decreasing the wear rate [3]. Inclusion of nano size Silica in the PEEK showed the increase in the hardness than that of unfilled PEEK. The increase in the wt. \% of the silica showed the reduction in the elongation property of the PEEK and reduction in strength due to pre matured failure. Degree of crystallinity can be increased by addition of silica in limited amount [4,5]. Mixing of AlN in micro size and nano size ceramic particles leads to the increase in the micro hardness. It was also seen that hardness is greater for nano composites than micro composites due lesser distance between in the nano particles [6]. Higher thermal stability was observed by Thermo 
gravimetric analysis (TGA) of Zircornia PEEK composites at high temperature as compared to the unfilled PEEK. Zirconia did not affect the glass transition temperature but the hardness, tensile strength, tensile and flexural modulus showed an increase [7]. HA-PEEK composite shows exponential increase in the Young's modulus. Tensile strength of the HA-PEEK decreases after the wt.\% is higher than 10 in a linear way. The tensile strength of HA-PEEK composite is near to cortical bone and even the strain to values is similar to cortical bone. The results have enhanced the research in the field of HA-PEEK bio-composite [8]. Composite of PEEK and $\mathrm{Si}_{3} \mathrm{~N}_{4}$ has also shown the similar changes in the mechanical properties. Increasing the amount of $\mathrm{Si}_{3} \mathrm{~N}_{4}$ in the PEEK leads to the linear increment in the hardness, compressive strength and bending strength but it shows reduction above $1.25 \%$ weight. Coefficient of friction reduces up to the $7.5 \mathrm{wt}$ \% then tends to increase [9]. PEEK$\mathrm{Al}_{2} \mathrm{O}_{3}$ composites micro hardness and stiffness rises with the rise in the content of $\mathrm{Al}_{2} \mathrm{O}_{3}$. Friction coefficient was found higher than unfilled PEEK. Wear resistance reduces above 3.5 vol\% of $\mathrm{Al}_{2} \mathrm{O}_{3}$ [10]. PEEK reinforced with Short carbon fiber has shown a significant increase in tensile strength, if the mass fraction is greater than $10 \%$ the rate of increase of tensile strength reduces. SCF reinforcement increases the thermal expansion of the composite [11]. Incorporation of carbon fiber reduces ductility and improves wear resistance [12]. When CF was added In the PEEK matrix the machinability of composite decreased as compared to the pure PEEK. Mechanical properties are not uniform for CF/PEEK it totally depends on the distribution of carbon fiber in the PEEK matrix [13, 14]. Polytetrafluroethylene addition in the PEEK has showed its improvement in the performance. A high improvement was seen in both wear and frictional coefficient. Only increase was seen in the case of Impact Strength rest all the strength and modulus decreased with increase amount of PTFE in the PEEK [15]. The inclusion of the glass fiber in the PEEK increased the tensile strength, tensile modulus, flexural modulus of the composite compared to the unfilled pure PEEK and the wear resistance against the sliding material [16]. PEEK when filled with inorganic materials like $\mathrm{SCF}, \mathrm{TiO}_{2}, \mathrm{ZnS}$, Graphite, $\mathrm{SiO}_{2}$ leads to moderate increase in the crystalline structure of the composites, matrix hardness, stiffness and little increase in the tensile strength. Mechanical properties depend on the fiber orientation in the matrix [17]. It was found that frictional coefficient and specific wear are higher for dry condition than for water lubricated condition. Transferred films were removed due to the lubrications [18]. PEEK wear rates depends on the temperature condition on the operation is performed [19].

\section{CONCLUSION}

Reinforcement of the various material may one way to enhance the mechanical property of PEEK. The structural change may the other process to produce the satisfied mechanical behavior. So selection of proper composition and correct structured material is a very important factor of the PEEK composite to produce prominent results.

\section{REFERENCES}

[1] Petropoulos G.; Mata F.; Paulo .Davim J.; Satistical study of surface roughness in turning PEEK.Materials and Design 29 , 2008, pp-218223.

[2] Goyal R.K.; Tiwari A.N.; Mulik U.P; Negi Y.S.; Journal Applied Polymer Science, 2007,pp-104-568.

[3] Wang Q.H.; Xu J.; Shen W.C.; Xue Q.; The effect of nanometer SiC filler on the tribological behavior of PEEK, Wear 209,1997, pp-316321.

[4] Lai Y.H.; KuoM.C.; Huang J.C.; Chen M.; On the PEEK composites reinforced by surface-modified nano-silica, Material Science and Engineering A, vol- 458, 2007, pp-158-169.

[5] Kuo M.C.; Tsai C.M.; Huang J.C.; Chen M.; PEEK composites reinforced by nano-sized $\mathrm{SiO}_{2}$ and $\mathrm{Al}_{2} \mathrm{O}_{3}$ particulates, Materials Chemistry and Physics 90, 2005, pp-185-195.

[6] Goyal R.K.; Negi Y.S.; Tiwari A.N.; Micro hardness of PEEK/ceramic micro-and nano composites: Correlation with Halpin -Tsai model, Material Science and Engineering A, vol-491, 2008, pp- 230-236.

[7] Mishra T.K.; Kumar A.; Verma V.; Pandey K.N.; Kumar V.; PEEK composites reinforced with nano filler, Composite Science and Technology, vol-72, 2012, pp-1627-1631.

[8] Abu Bakar M.S.; Cheang P.; Khor K.A.; Mechanical properties of Injection Molded hydroxyapatite-polyetheretherketone, Composite Science and Technology, vol-63, 2003, pp-421-425.

[9] Wang Q.H.; Xu J.; Shen W.C.; Lie W.; An investigation of the friction and wear properties of nanometer $\mathrm{Si}_{3} \mathrm{~N}_{4}$ filled PEEK, Wear 196, 1996, pp-82-86.

[10] Goyal,R.K; Negi, Y.S; Tiwari, A.N; Mulik.U.P; Dynamic Mechanical Properties of Al2O3/Poly(ether ether ketone)Composites.Journal of Applied Polymer Science, Vol. 104,2007, 568-575. http://dx.doi.org/10.1002/app.25607

[11]Zhang.L; Li M; Study on properties of PEEK composites reinforced by SCF. Advance Material Research Vol 476-478, 2012, pp-705-709.

[12] Zhang J.; Cai C.; Friction and wear properties of carbon fiber reinforced PEEK composite under water lubrication, Advance Mechanics Material, Vol-66-68, 2011, pp-1051-1054.

[13] Ji Shijun.; Sun C.; Ji Zhoa.; Yu H.; Study about Mechanical Property and Machinabilty of Carbon-Fiber Reinforced Polyetheretherketone, Journal of Computational and Theoritical Nanoscience, Vol- 12, 2015, pp- 2969-2973.

[14] Ji Shijun.; Sun C.; Ji Zhoa.; Liang Fusheng.; Comparision and Analysis on Mechanical Property and Machinabilty about Polyetheretetherketone and Carbon Reinforced Polyetheretherketone, Materials, 2015, vol-8, pp-4118-4130.

[15] Bijwe J.;Sen S.; Ghosh A.; Influence of PTFE content in PEEK-PTFE blends on mechanical properties and tribo-performance in various wear modes, Wear 258, 2005,1536-1542. http://dx.doi.org/10.1016/j.wear.2004.10.008

[16] Li E.Z.; Guo W.L.; Wang H.D.; Xu B.S.; Liu X.T.; Research on Tribological Behaviour of PEEK and Glass Fiber Reinforced PEEK Composite, Physics Procedia, vol-50, 2013, pp- 453-460.

[17] Lin L. Y.; Tlatlik H.; Gralla R.; Igartua M.A.; P de. Baets. de P.; Schlarb A.K.; Mechanical and Thermal behaviours Polyetheretherketone-based multi-scaled composites, Journal of Composites Materials 47(17), pp2087-2096.

[18] Mimaroglu A.; Unal H.; Ozel A.; Tribological Performance of Polyetheretherketone and its Composites under Water environment, Macromol. Symp., vol-327,2013,pp-108-113.

[19] Rzatki F. D.; Barboza D. V. D.; Schroeder R. M.; Barra G. M. D. O.; Binder C.; Klien A. N.; De Mello. J. D. B.; Effect of temperature and atmosphere on the tribological behavior of Polyetheretherketone composite, Friction, vol-3(4), 2015, pp-259-265. 